\title{
User-Based Channel Assignment Algorithm in a Load-Balanced IEEE 802.11 WLAN
}

\author{
Mohamad Haidar, Ecole de Technolgie Superieure, Canada \\ Hussain Al-Rizzo, University of Arkansas at Little Rock, USA \\ Yupo Chan, University of Arkansas at Little Rock, USA
}

Robert Akl, University of North Texas, USA

\begin{abstract}
A new load balancing algorithm is presented based on power management of Access Points (APS) to reduce congestion at hot spots in Wireless Local Area Networks (WLANs) and to assign channels to APs The algorithm first finds the Most Congested Access Point (MCAP), then decreases its transmitted power in discrete steps, and the process continues until the users'assignment which leads to a high balance index is reached. A new mathematical programming formulation is then applied to assign channels to the APs such that the Signal-to-Interference Ratio (SIR) at the users'level is maximized. Results show that the algorithm is capable of reducing the overall congestion at hot spots in a WLAN and increases the SIR significantly for cases involving relatively large WLANs. In the process, network throughput is increased. [Article copies are available for purchase from InfoSci-on-Demand.com]
\end{abstract}

Keywords: Access Point; Channel Assignment; Congestion; Load Balancing; Signal-to-Interference Ratio; Wireless Local Area Network

\section{INTRODUCTION}

More industries, organizations, and offices are installing WLANs to support the growing demand of wireless users. The motivation is to reduce the cost of running cables and, more importantly, meet the demand of users who wish to stay connected to the network, communicate with others, and access the World Wide Web while roaming.

Numerous research has been conducted on load balancing (Akl \& Park, 2005; Fang \& Low, 2004; Haidar, et al., May 2007; Papanikos \& Logothetis, 2001; Velayos, et al., 2004) and channel assignment (Akl \& Arepally, 2007; Eisenblätter, et al., 2007; Haidar, et al., Sept 2007; Kulkrani \& Shenoy, 2004; Lee, 
et al., 2002; Mishra, et al., 2005) separately. The authors are aware of only one research that involved combining load balancing and channel assignment (Mishra, et al., 2006). The advantage of combining load balancing and channel assignment in one algorithm is to increase network throughput by utilizing available resources efficiently.

In the load balancing related literature, the authors of (Akl \& Park, 2005; Fang \& Low, 2004) proposed minimizing AP congestion in WLANs using an Integer Linear Programming (ILP) formulation. In (Akl \& Park, 2005; Fang \& Low, 2004), the load at the MCAP was minimized once without applying power management. A free space propagation model is used in (Akl \& Park, 2005; Fang \& Low, 2004) to associate demand points with candidate APs. The authors in (Papanikos \& Logothetis, 2001) proposed a load balancing technique that allows a wireless station to join an AP depending on the number of existing users and the mean Received Signal Strength Indicator (RSSI). In (Velayos, et al., 2004), the authors proposed a load-balancing scheme for overlapping wireless cells. Load Balancing Agents (LBA) running at each AP broadcast periodically the local loads via the Ethernet backbone and determine whether the AP is overloaded, under-loaded, or balanced. Users in overloaded APs are forced to dissociate from their corresponding AP and associate with an under-loaded AP. In (Haidar, et al., May 2007), we proposed an algorithm that decrements the transmitted power at the MCAP in discrete steps until any of the three following conditions is met: (1) at least one user can no longer associate with any potential AP, (2) the desired user's data rate can no longer be accommodated, or (3) the balance index value exceeds a predefined threshold. At that stage, the network's load is distributed efficiently over the network compared to traditional association based on the highest RSSI. This is accomplished with a lower transmitted power levels at the MCAPs leading to less co-channel and adjacent channel interferences.

At the planning stage, channel assignment is often considered in the context of the problem of AP placement (Eisenblätter, et al., 2007; Kulkrani \& Shenoy, 2004; Lee, et al., 2002). In (Lee, et al., 2002), an algorithm was proposed for channel assignment in hot-spot service areas using an ILP formulation. The objective was to avoid assigning non-overlapping channels among neighboring APs, leaving other available channels unutilized. The authors in (Eisenblätter, et al., 2007) noted that previous AP placement and channel assignment were always designed sequentially. An integrated model that addresses both issues concurrently was proposed. It was shown that through an ILP formulation, AP placement and channel assignment could be combined, resulting in better performance. In (Kulkrani \& Shenoy, 2004), the authors presented a greedy heuristic algorithm that provides maximum coverage while minimizing interference in the overlapping APs. A channel-assignment based on a Non-Linear Integer Program (NLIP) that minimizes channel interference among neighboring APs was provided in (Akl \& Arepally, 2007). The authors in (Mishra, et al., 2005) proposed a weighted variant of the coloring-graph algorithm to improve the usage of wireless spectrum in WLANs. In (Haidar, et al., Sept 2007), we proposed a channel assignment algorithm, based on minimizing interference between APs. The algorithm was invoked after the load has been balanced based on the power management scheme presented in (Haidar, et al., May 2007). Results indicate that a different channel assignment is required to meet users' demands. Note that the channel assignment at the planning stage may result in severe interferences due to users' distribution-even after power has been adjusted on APs. Finally, the authors in (Mishra, et al., 2006) also proposed a client-based channel assignment and load balancing approach that lead to better usage of the wireless spectrum. They showed that a joint solution has significant advantages, such as capturing the effect of interference at the clients and a better channel re-use. Since the authors did not employ any specific indoor propagation model, their model was based on minimizing channel conflicts between APs within one-hop distance from each other, as- 
suming all APs transmit at the same power. However, in our case, the coverage, which is directly proportional to the transmitted power at the AP, is not fixed after a load-balanced state is achieved. We tend to calculate interferences at users that even extend beyond one-hop distance APs. Losses due to signal propagation are also accounted for.

In this article, we extend our research reported in (Haidar, et al., May 2007; Haidar, et al., Sept 2007) by formulating a NLIP model to maximize the total SIR at the user level, taking into account adjacent and co-channel interference. The authors in (Boulmalf, et al., 2005) showed that an increase in the SIR of the network would be reflected in an increase in the network throughput. After the network achieves a balanced state, as measured by the balance index (Balachandran, et al., 2002; Chiu, et al., 1989), the SIR of all users is used to reassign channels to the APs. The algorithm in (Haidar, et al., May 2007) balances the load on the WLAN in order to utilize the available bandwidth more efficiently. This is accomplished by reassigning users to different APs, while decrementing the transmitted power of the MCAPs. After achieving a balanced-load state, channels are assigned to APs based on minimizing the interference among neighboring APs ((Haidar, et al., Sept 2007). Although assigning channels after load balancing provided better results than assigning channels without load balancing, it still does not take advantage of the users' presence on the network to improve the channel assignment. Therefore, we propose another channel assignment that leads to the maximum SIR for the users. In other words, channels assigned are determined by the user's SIR rather than interference between neighboring APs. One advantage of such a scheme is that if a certain "assigned" channel suffers frequency-selective fading, the effect is recorded at the user(s) affected in terms of a sudden drop in their SIR values below a certain threshold. This triggers the central server to run the algorithm again in order to assign different channels based on maximizing the SIR.
The remainder of this article is organized as follows. The power management algorithm is presented in the next section. The channel assignment algorithms are presented in the section after, followed by the summary of the proposed algorithm and the assumptions involved. Then, the numerical results are presented, followed by the conclusion.

\section{THE POWER MANAGEMENT ALGORITHM}

The power management algorithm is based on iteratively decrementing the transmitted power at the MCAPs in discrete steps. The received power at each user's location is evaluated using the No Line of Sight (NLOS) Path Loss model (Lei, et al., 2005):

$$
\begin{aligned}
& P L(d)=P L_{0}+29.4 \mathrm{~L} \mathrm{og} 10(d)+ \\
& 6.1 x_{\alpha} \log 10(d)+2.4 y+1.3 x y \\
& \left|x_{\alpha}\right| \leq 1.3 \\
& \left|x_{s}\right| \leq 1.5 \\
& |y| \leq 1.5
\end{aligned}
$$

Here, $P L_{0}$ is the free-space path-loss in $\mathrm{dB}, d$ is the distance between user $i$ and $A P_{j}$ in meters, and $x_{\alpha}, x_{s}$, and $y$ are mutually independent Gaussian random variables of zero mean and unit variance.

Once the power received at a user from an AP exceeds the receiver's predefined sensitivity threshold, that user becomes a candidate for association with that AP. Thus, a user can be a candidate for association with several APs.

The WLAN under consideration consists of a grid of $M$ APs distributed in a single-floor indoor environment. A set of $N$ randomly distributed users seek to associate with an AP each. A user is defined by its randomly assigned position and data rate. After the initial channel assignment, which is based on minimizing the interference between neighboring APs, we seek 
to redistribute users' associations in order to minimize the overall congestion in the network. We then assign channels to APs based on the final association of users to APs.

As mentioned, this is achieved by first identifying the MCAP and decrementing its transmitted power in discrete steps. This is done such that each user is associated with one and only one AP. The congestion factor at $A P_{j}, C_{j}$ ,is defined as:

$C_{j}=\frac{\sum_{i=1}^{N_{j}} R_{i}}{B W_{j}}$,

where $N_{j}$ is the number of users associated with $A P_{j}, R_{i}$ is the data of user $i$, and $B W_{j}$ is the maximum bandwidth for each AP (54 Mbps for IEEE $802.11 \mathrm{~g}$ ). The commercial software package LINGO (www.lindo.com) is used to solve the following NLIP (Haidar, et al., May 2007).

$\min$

$$
\max \left\{C_{1}(x), C_{2}(x), \ldots, C_{M}(x)\right\}
$$

subject to,

$$
\sum_{j=1}^{M} x_{i j}=1
$$

$C_{j}(x)=\frac{\sum_{i=1}^{N_{j}} R_{i} \cdot x_{i j}}{B W_{j}}$,

for $j \in\{1, \ldots, M\}$.

Objective (3) minimizes the congestion at the MCAP in each iteration. Constraint (3.1) states that each user must be assigned to one and only one AP at any time. The binary variable, $x_{i j}$, is 1 when user $i$ is assigned to $A P_{j}$ and 0 otherwise. Constraint (3.2) defines the congestion factor at the APs as a function of the assignment.

It should be noted that as the users' associations are changing due to the decrease of the transmitted power at the MCAP, the algorithm appropriately relocates the new MCAP at each iteration based on the new bandwidth utilization $\left(C_{j}\right.$ 's of all APs), and decrements its power assuming no changes are occurring in the channel environment during the course of simulation. In other words, users' data rates suffer minimal fluctuations and the average data rate is considered constant over the simulation time, which depends on the variables involved and computer processing time. The final solution provides the power level of the individual APs and the final users' associations such that each user is connected to one AP.

\section{THE CHANNEL ASSIGNMENT ALGORITHMS}

In IEEE $802.11 \mathrm{~b} / \mathrm{g}$ WLAN, there exists 14 channels. Channels 1, 6 and 11 are non-overlapping, as shown in Figure 1. Only 11 channels are used in the US. Each channel spreads over $22 \mathrm{MHz}$ due to the Direct Sequence Spread Spectrum (DSSS) technique employed by IEEE $802.11 \mathrm{~b} / \mathrm{g}$. For instance, channel 1 ranges from $2.401 \mathrm{GHz}$ to $2.423 \mathrm{GHz}$ and its center frequency is $2.412 \mathrm{GHz}$. The center frequency of two adjacent channels is separated by 5 $\mathrm{MHz}$. The overlapping channel interference factor $w_{j k}$ is defined as follows (Haidar, et al., Sept 2007):

$w_{j k}=\left\{\begin{array}{cl}1-\left|C h_{j}-C h_{k}\right| \times c & \text { if } w_{j k} \mathbb{Q} \\ 0 & \text { otherwise }\end{array}\right.$,

where $C h_{j}$ is the channel assigned to $A P_{j}, C h_{k}$ is the channel assigned to $A P_{k}$ and $c$ is the nonoverlapping portion of two adjacent channels, expressed as a fraction of the frequency spectrum of a channel. For instance, channel 1 and channel 2 do not overlap from $2.401 \mathrm{GHz}$ to $2.406 \mathrm{GHz}$, as shown in Figure 1. Normalizing the overlap of $5 \mathrm{MHz}$ over the spectrum of 22 $\mathrm{MHz}, c$ is approximately equal to $1 / 5$. When the channels are far apart, as is the case with channels 1 and 6, $w_{j k}=0$ (i.e., no interference). When the two channels are the same, $w_{j k}=1$ 
Figure 1. The three non-overlapping channels

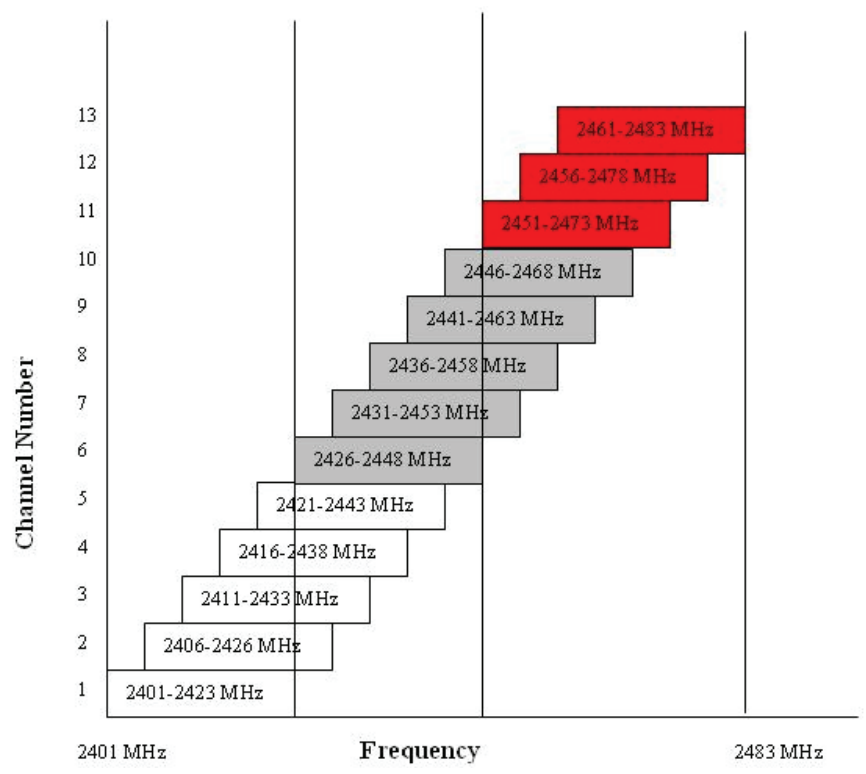

(i.e., maximum interference). Channels should be assigned to APs such that overlapping channel interference is minimized. However, there are limited channel resources (11 channels in IEEE $802.11 \mathrm{~b} / \mathrm{g}$ ). If the same channel is to be assigned to two or more APs that are located far enough from each other, the overlapping channel interference signal detected by each AP should be less than a given threshold.

We present two channel-assignment algorithms, to be executed in sequence. The first is commonly used to assign channels to APs in the planning stage, prior to users' connection in the network. In this algorithm, the objective is to minimize the total interference among neighboring APs. The second assigns channels to APs when users enter the network. Remember the objective of the algorithm developed in this article is to maximize SIR at the user level. The following variables are defined for both algorithms:

- $\mathrm{A}_{\mathrm{j}}$ is the set of neighboring APs to $A P_{j}$.

- $K$ is the number of available channels, 11 in IEEE $802.11 \mathrm{~b} / \mathrm{g}$.
- $D_{j k}$ is the distance between $A P_{j}$ and $A P_{k}$.

- $Q_{j}$ is the cardinality of set $\mathrm{A}_{\mathrm{j}}$

- $\quad P_{i k}$ is the power received by user $i$ associated with $A P_{k}$.

- $\quad P_{i j}$ is the power received by user $i$ from the interfering $A P_{i}$.

- $\operatorname{Interf}_{j k}$ is the interference on $A P_{j}$ from $A P_{k}$.

- $\quad I_{i j}$ is the total interference experienced by user $i$ due to all other APs $j$ (where $j \neq k$ ).

- $P_{k}$ is the power transmitted from $A P_{k}$.

\section{Channel Assignment Algorithm 1}

The mathematical formulation of the first channel assignment algorithm, based on minimizing interference between APs, is given by the following NLIP formulation:

$\min$ for all $j$

subject to

Interfjk $=\frac{w_{j k} P k}{P L\left(D_{j k}\right)}$ 
$w_{j k}=\left\{\begin{array}{cl}1-\left|C h_{j}-C h_{k}\right| \times c & \text { if } w_{j k} \notin \\ 0 & \text { otherwise }\end{array}\right.$

for $k \in \mathrm{A}_{\mathrm{j}}$, for $j \in\{1, \ldots, M\}$, for $C h_{j}, C h_{k} \in\{1, \ldots, K\}$.

Objective (5.1) minimizes the total interference at each $A P_{j}$. Constraint (5.2) defines the interference experienced by $A P_{j}$ due to neighboring $A P_{k}$. Constraint (5.3) is the overlapping channel interference factor $w_{j k}$. The NLIP determines the integer variables $C h_{j}$ and $C h_{k}$ corresponding to the assignment of channels to APs. This algorithm has been used in (Akl and Arepally, 2007; Haidar, et al., Sept 2007) to assign channels in the planning stage, before user connections exist.

\section{Channel Assignment Algorithm 2}

The mathematical-programming formulation of the second channel assignment algorithm, based on maximizing the SIR for the users, is given below as an NLIP:

$\max \sum_{i=1}^{N} \sum_{j=1}^{M} \operatorname{SIR}_{i j}(k)$ for all $k$, where $k \neq j$,

subject to $w_{j k}=\max \left(0,1-\left|C h_{j}-C h_{k}\right| \times c\right)$

$I_{i j}=\sum_{i=1}^{N} \sum_{j=1}^{M}\left(P_{i j} \bullet w_{j k}\right), k \neq j$,

$\operatorname{SIR}_{i j}(k)=\frac{P_{i k}}{I_{i j}}, \forall i, j, j \neq k$

for $j, k \in\{1, \ldots, M\}$,

for $i \in\{1, \ldots, N\}$, for $C h_{j}, C h_{k} \in\{1, \ldots, K\}$.

Objective (6.1) maximizes the total SIR for all $N$ users. The total SIR refers to the sum of all SIR values at the individual users in the network. Constraint (6.2) is the overlapping channel interference factor. Constraint (6.3) defines the interference at user $i$ due to all APs $j$ except $A P_{k}$ to which user $i$ is connected. Constraint (6.4) defines the SIR at user $i$. The NLIP determines the integer variables $C h_{j}$ and $C h_{k}$ corresponding to the assignment of channels to APs.

It should be emphasized that the scenarios presented in this article demonstrate the improvements achieved by combining our load balancing algorithm based on power management with the proposed channel assignment at the user level as compared to the existing approaches based solely on interference between APs. If the algorithm is to be executed in real time, each user $i$ must update the serving $A P_{k}$ with its associated $\operatorname{SIR}_{i}(k)=\sum_{j} \operatorname{SIR}_{i j}(k)$ upon registering with it. Then each AP, synchronized with the other APs, periodically requests SIR from its users to be transferred to a central server where the proposed algorithm would be running. Similarly, in case of a change in the current user distribution, resulting from users joining or exiting the network, the APs would also transfer the $\operatorname{SIR}_{i j}(k)$ information to the central server that runs the load balancing and channel assignment algorithm to reassign channels to the APs.

One can think of our model as representing the scenario in a time slice, for a particular user distribution. It is important to note that userto-user interference was assumed negligible due to its low transmitted power compared to the AP's transmitted power. The model can be executed for all time slices sequentially in which the $\operatorname{SIR}_{i j}(k)$ information is updated for each time slice.

\section{SUMMARY OF THE PROPOSED ALGORITHM}

The 11-step algorithm, which assigns channels in a power managed WLAN, is summarized as follows: 
1. Assign channels to the M APs based on the NLIP model in (5), which is based on minimizing the total interference between APs.

2. Generate $\mathrm{N}$ randomly distributed users i.

3. Compute the RSSI at each user i from each $\mathrm{AP}_{\mathrm{j}}$ using the path loss model in (1).

4. Generate a binary matrix that assigns 1 in the corresponding $(i, j)$ if a user's RSSI exceeds a given threshold value or 0 otherwise.

5. Solve the user-AP assignment NLIP, using the objective function and constraints defined in (3), (3.1), and (3.2).

6. Identify the new MCAP.

7. Decrement the power level of the new MCAP by a discrete step size of $1 \mathrm{dBm}$.

8. Repeat step 7 until at least one user can no longer associate with an AP.

9. Compute the interference caused by neighboring APs to each user.

10. Compute the SIR for each user.

11. Reassign channels to the APs by invoking the model in (6). If a different user distribution is deployed, go back to step 3 .

Note that the following assumptions are imposed in the course of the simulations described in the next section:

1. AP coordinates are given.

2. The average data rate of each user is considered constant during the simulation period.

3. User-to-user interference is ignored.

4. Users are stationary.

5. User-AP association is assumed to be fixed during each iteration until a new association is obtained in step 4.

6. Receiver sensitivity is $-85 \mathrm{dBm}$.

7. Receiver detection threshold is -110 $\mathrm{dBm}$.

8. Initial transmitted power of each AP is 20 $\mathrm{dBm}$.

Another key metric, in addition to the congestion factor $\left(\mathrm{C}_{j}\right)$, was used to quantify how balanced is the network and determine the convergence of the load balancing algorithm. This metric is referred to as the balance index, which appeared for the first time in (Balachandran, et al., 2002; Chiu, et al., 1989). The balance index reflects the capacity used in each AP. Let $T_{j}$ be the total traffic of $A P_{j}$. Then the balance index $\beta$ is:

$\beta=\frac{\left(\sum T_{j}\right)^{2}}{\left(n \times \sum T_{j}^{2}\right)}, j \in\{1, \ldots, M\}$

where $n$ is the number of cells with overlapped coverage area. Thus, when an average user can tie into multiple APs, $n$ becomes large. With this definition, the denominator is the smallest when all the $R_{j}$ are equal, and there is no overlap in coverage ( $n$ is small). It assumes its largest value when $R_{j} \mathrm{~s}$ are disparate, and there is excess overlap in service areas ( $n$ is large). The balance index has the property that it approaches 1 when each AP has its equal share of user traffic. Conversely, it gets closer to a small fraction (such as $1 / n$ ) when $n$ is large and there is a huge disparity among $R_{j} \mathrm{~s}$, leading to the situation that APs are heavily unbalanced.

\section{NUMERICAL RESULTS}

In order to validate the algorithm proposed in this article, we initially considered two benchmark problems. In the first, all users are distributed in the overlapping region between APs, as shown in Figure 2. In this case, the best channel assignment leading to the maximum SIR among users is the same as that leading to minimum interference between APs. This is obvious since all users fall in the overlapping coverage region between the two APs.

In the second, all the users are in the corresponding AP coverage zone with none in the overlap region, as shown in Figure 3. In this case, the best channel assignment, based on maximizing the SIR, could be any set of combinations of channels since the users are not in the common overlapping region and adjacent channel or co-channel interference 


\section{Figure 2. Users in the overlapping region}

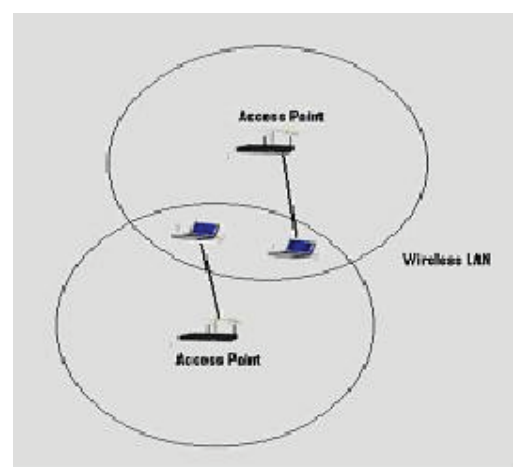

Figure 3. Users not in the overlapping region

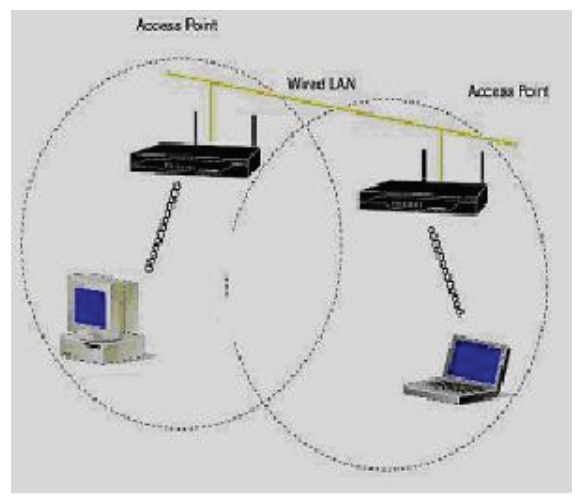

has no impact at the users. Therefore, in this case, even the same channel can be assigned to all APs. These observations were validated by our algorithm when it was run under these two conditions.

Next, the scalability of the algorithm is verified by applying it to scenarios with different numbers of APs and users. In each scenario, we compare the results of our algorithm to previously published algorithms. It is important to mention that the convergence time of any of the scenarios presented ranged from few seconds to few minutes based on meeting any of the aforementioned three conditions that terminate the simulation:

1. At least one user can no longer associate with a candidate AP.
2. A particular user's data rate can no longer be accommodated.

3. The balance index value exceeds 0.97 .

The simulations were carried out with service areas consisting of 4, 6, 9 and 12 APs and 20, 30, 45 and 60 users, respectively. APs are placed 60 meters from each other, 20 meters from adjacent walls and the service area's lengths and widths vary with the number of APs.

\section{Scenario 1: 4 APs and 20 Users}

In scenario 1 , we consider a grid of 4 APs over a $100 \mathrm{~m} \times 100 \mathrm{~m}$ area and 20 randomly distributed users as shown in Figure 4. We generate a binary matrix based on a receiver's sensitivity threshold of $-85 \mathrm{dBm}$, as shown in Table 1 .

As can be seen from Table I, a user can be a candidate for association with multiple APs if its signal level exceeds the threshold value. For instance, user $1, U_{l}$, can connect to $A P_{l}$, $A P_{2}$, and $A P_{3}, U_{10}$ can connect to $A P_{2}$ and $A P_{4}$, and $U_{19}$ can connect only to $A P_{3}$.

We ran the 11-step algorithm until step 8, right after the power management step. This yields the final power level at each AP, which in turn leads to the final received power at the users, and the final association matrix of users. The final power level map and the user-AP associations' matrix are depicted in Figure 5 and

Figure 4. Power level map for scenario 1 at ground level (0 meters)

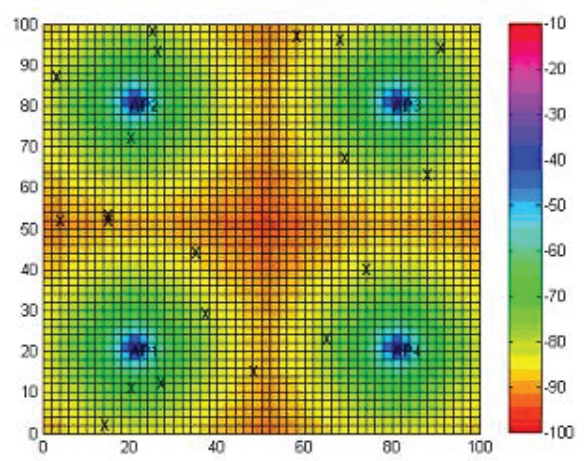


74 Int. J. of Interdisciplinary Telecommunications and Networking, 1(2), 66-81, April-June 2009

Table 1. Initial associations' matrix (scenario 1)

\begin{tabular}{|l|l|l|l|l|}
\hline Users & AP1 & AP2 & AP3 & AP4 \\
\hline U1 & 1 & 1 & 1 & 0 \\
\hline U2 & 0 & 0 & 1 & 0 \\
\hline U3 & 0 & 0 & 0 & 1 \\
\hline U4 & 0 & 0 & 0 & 1 \\
\hline U5 & 1 & 1 & 1 & 1 \\
\hline U6 & 1 & 1 & 0 & 0 \\
\hline U7 & 0 & 1 & 0 & 0 \\
\hline U8 & 0 & 0 & 1 & 1 \\
\hline U9 & 0 & 0 & 1 & 0 \\
\hline U10 & 0 & 1 & 0 & 1 \\
\hline U11 & 1 & 0 & 1 & 0 \\
\hline U12 & 0 & 0 & 1 & 1 \\
\hline U13 & 1 & 0 & 0 & 0 \\
\hline U14 & 0 & 1 & 1 & 1 \\
\hline U15 & 1 & 1 & 0 & 1 \\
\hline U16 & 1 & 0 & 1 & 0 \\
\hline U17 & 1 & 1 & 0 & 0 \\
\hline U18 & 1 & 1 & 0 & 0 \\
\hline U19 & 0 & 0 & 1 & 0 \\
\hline U20 & 1 & 1 & 0 & 0 \\
\hline
\end{tabular}

Figure 5. Power level map for scenario 1 after power adjustment

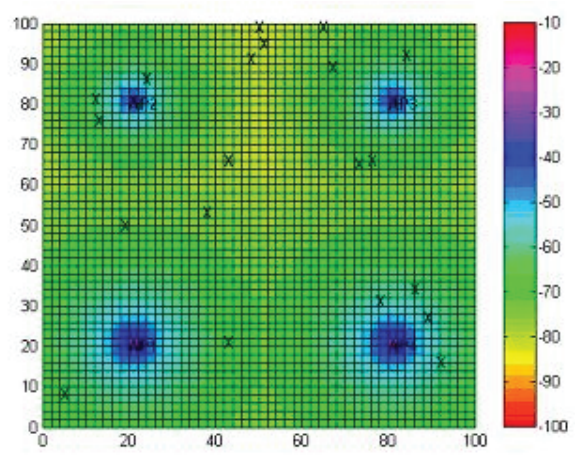

Table 2, respectively. At the end of step 8, the transmitted power was dropped on $A P_{1}$ from 20 $\mathrm{dBm}$ to $11 \mathrm{dBm}, A P_{2}$ to $9 \mathrm{dBm}, A P_{3}$ to $4 \mathrm{dBm}$
Table 2. Final users' assignment with power management (Scenario 1)

\begin{tabular}{|l|l|l|l|l|}
\hline Users & AP1 & AP2 & AP3 & AP4 \\
\hline U1 & 0 & 1 & 0 & 0 \\
\hline U2 & 0 & 0 & 1 & 0 \\
\hline U3 & 0 & 0 & 0 & 1 \\
\hline U4 & 0 & 0 & 0 & 1 \\
\hline U5 & 0 & 1 & 0 & 0 \\
\hline U6 & 1 & 0 & 0 & 0 \\
\hline U7 & 0 & 1 & 0 & 0 \\
\hline U8 & 0 & 0 & 0 & 1 \\
\hline U9 & 0 & 0 & 1 & 0 \\
\hline U10 & 0 & 1 & 0 & 0 \\
\hline U11 & 0 & 0 & 1 & 0 \\
\hline U12 & 0 & 0 & 0 & 1 \\
\hline U13 & 1 & 0 & 0 & 0 \\
\hline U14 & 0 & 0 & 1 & 0 \\
\hline U15 & 0 & 0 & 0 & 1 \\
\hline U16 & 1 & 0 & 0 & 0 \\
\hline U17 & 1 & 0 & 0 & 0 \\
\hline U18 & 0 & 1 & 0 & 0 \\
\hline U19 & 0 & 0 & 1 & 0 \\
\hline U20 & 0 & 1 & 0 & 0 \\
\hline
\end{tabular}

Figure 6. User to AP association

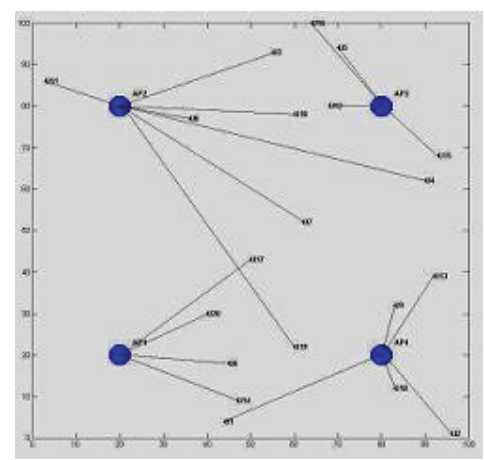

and $\mathrm{AP}_{4}$ to $3 \mathrm{dBm}$. Table II confirms that each user is associated with one AP, which is the first constraint in the NLIP model (3). Figure 6 depicts such a user-AP association. 
Table 3 displays the Congestion Factor (CF) for each AP and the balance index for three cases: No Power Management (NPM) where each user connects to the AP with the highest RSSI, the CF for each AP based on the ILP model (3), where only the load at the initial MCAP is reduced but NPM, and the CF achieved at each AP by our proposed Power Management (PM) algorithm.

As can be seen from Table 3, the NLIP model (3) by itself was able to reduce initial congestion at $A P_{1}$ only. However, the proposed PM algorithm was able to share the load more evenly across the entire network. This also can be seen through the values of the balance index, which clearly indicates that our proposed algorithm leads to the most balanced load. Finally, we proceeded with our algorithm through steps 9 to 11 to assign channels to the APs based on maximizing the SIR at the users. Table IV documents the initial channel assignment, based on minimizing AP interference with NPM, and channel assignment based on maximizing the SIR at the user level after PM, based on model (6).

Table 4 shows that if we were to start with a channel assignment in the planning stage and keep that channel assignment unchanged after users enter the network, the average SIR would be 4.48 per user. However, by applying our algorithm at the balanced state, the average SIR, expressed as a dimensionless ratio, was increased to 5.83 , which corresponds to $30 \%$

Table 3: comparison between congestion factors (scenario 1)

\begin{tabular}{|l|l|l|l|}
\hline & CF : NPM & $\begin{array}{l}\text { CF Model } \\
\text { (3): NPM }\end{array}$ & $\begin{array}{l}\text { CF with } \\
\text { PM }\end{array}$ \\
\hline AP1 & 0.7323 & 0.5416 & 0.4404 \\
\hline AP2 & 0.4735 & 0.5378 & 0.4155 \\
\hline AP3 & 0.2283 & 0.3547 & 0.4559 \\
\hline AP4 & 0.2393 & 0.2393 & 0.3615 \\
\hline $\begin{array}{l}\text { Balance } \\
\text { Index( }(\boldsymbol{)})\end{array}$ & $80.47 \%$ & $91.43 \%$ & $99.27 \%$ \\
\hline
\end{tabular}

improvement. The average SIR is defined as the total SIR of the users divided by the number of users in the network.

\section{Scenario 2: 6 APs and 30 Users}

In scenario 2, we considered 6 APs over 160 $\mathrm{m} \times 100 \mathrm{~m}$ and 30 randomly distributed users. We ran the 11-step model until step 8 to get the final transmitted power level at each AP and the final user-APassociation. The transmitted power remained the same on $A P_{1}$ and $A P_{2}$, whereas the power of $A P_{3}$ dropped to $18 \mathrm{dBm}, A P_{4}$ to 9 $\mathrm{dBm}, A P_{5}$ to $-1 \mathrm{dBm}$ and $A P_{6}$ to $18 \mathrm{dBm}$. Table 5 summarizes the $\mathrm{CF}$ for each AP.

Again, the NLIP (3) by itself was not able to equalize the load at the APs. It is interesting to note that the $\mathrm{CF}$ of $A P_{2}$ is very low because users associated with it happened to be at a short distance such that their RSSI from other APs are considered to be noise and there is no way they can associate to another AP. Therefore, the following users will remain connected to their respective AP no matter how long we run the algorithm. The same applies to $A P_{l}$. Under the PM algorithm, the remaining APs achieved a more balanced load distribution (around 89\% balanced load distribution), compared with the default association and the NLIP model (3) by itself. It is noticed" that the algorithm converged before exceeding a balance index value of $95 \%$. This is because it was forced to quit based on the other two constraints (at least a

Table 4: comparison between model (5) and model (6) (scenario 1)

\begin{tabular}{|l|l|l|}
\hline & $\begin{array}{l}\text { Initial Channel } \\
\text { Assignment }\end{array}$ & $\begin{array}{l}\text { Final Channel } \\
\text { Assignment }\end{array}$ \\
\hline AP1 & 11 & 1 \\
\hline AP2 & 1 & 6 \\
\hline AP3 & 8 & 11 \\
\hline AP4 & 3 & 2 \\
\hline Avg. SIR & $\mathbf{4 . 4 8}$ & $\mathbf{5 . 8 3}$ \\
\hline
\end{tabular}


Table 5. Comparison between congestion factors (Scenario 2)

\begin{tabular}{|l|l|l|l|}
\hline & $\begin{array}{l}\text { CF } \\
\text { NPM }\end{array}$ & $\begin{array}{l}\text { CF Model (3): } \\
\text { NPM }\end{array}$ & CF with PM \\
\hline AP1 & 0.1279 & 0.1279 & 0.1880 \\
\hline AP2 & 0.0791 & 0.0791 & 0.0791 \\
\hline AP3 & 0.4296 & 0.4296 & 0.4194 \\
\hline AP4 & 0.5365 & 0.6034 & 0.4095 \\
\hline AP5 & 0.7556 & 0.6887 & 0.4419 \\
\hline AP6 & 0.2171 & 0.2171 & 0.5308 \\
\hline Balance Index ( $\beta)$ & $68.95 \%$ & $70.23 \%$ & $89.00 \%$ \\
\hline
\end{tabular}

user can no longer associate to an AP or a user's data rate can no longer be accommodated) and that is the best balance index achieved without violating the other constraints. We proceed to steps 9 through 11, assigning channels to the APs, the results of which are shown in Table 6. The average SIR was improved by almost 6\%. In this case, both $A P_{3}$ and $A P_{4}$ used channel 6, which means there were no users in their overlapping region.

\section{Scenario 3: $9 \mathrm{APs}$ and 45 Users}

In this scenario, we deploy 9 APs over $160 \mathrm{~m}$ $\times 160 \mathrm{~m}$ area. The APs are distributed in a 3 $\times 3$ grid with 45 users randomly distributed in

Table 6. comparison between model (5) and model (6) (scenario 2)

\begin{tabular}{|l|l|l|}
\hline & $\begin{array}{l}\text { Initial Channel } \\
\text { Assignment }\end{array}$ & $\begin{array}{l}\text { Final Channel } \\
\text { Assignment }\end{array}$ \\
\hline AP1 & 6 & 2 \\
\hline AP2 & 1 & 11 \\
\hline AP3 & 6 & 6 \\
\hline AP4 & 11 & 6 \\
\hline AP5 & 1 & 8 \\
\hline AP6 & 11 & 1 \\
\hline Avg. SIR & $\mathbf{2 . 6 4}$ & $\mathbf{2 . 8 0}$ \\
\hline
\end{tabular}

the service area. Results for the CF and channel assignment are depicted in Tables 7 and 8 , respectively.

Model (3) decreased the load at the MCAP $\left(A P_{3}\right)$ but increased it greatly on $A P_{8}$. Our PM algorithm distributed the load evenly (around 97\% balanced). This load distribution was achieved at the following power levels at the APs: $7 \mathrm{dBm}, 1 \mathrm{dBm},-4 \mathrm{dBm}, 2 \mathrm{dBm}$, $-5 \mathrm{dBm},-4 \mathrm{dBm}, 6 \mathrm{dBm},-3 \mathrm{dBm}$ and $2 \mathrm{dBm}$, respectively.

Table 8 shows that the average SIR of the network was improved to 1.93 (almost $74 \%$ improvement). This significant improvement can be attributed to the fact that after load distribution some users that were close to their original AP are now redirected to a farther AP that reduces the AP congestion. Therefore, a user may suffer interference from its nearest APs, yet have enough RSSI to associate with an AP other than the original AP.

\section{Scenario 4: 12 APs and 60 Users}

Finally, we apply our algorithm to a 12-AP service area. The 12 APs are deployed over a $3 \times 4$ grid. This time 60 users are generated randomly in the service area. Similar steps are followed for comparison between the channel assignment algorithm based on the minimum interference between APs and our proposed PM algorithm. Comparison of results is listed in Tables 9 and 10. 
Table 7. Comparison between congestion factors (scenario 3)

\begin{tabular}{|l|l|l|l|}
\hline & CF :NPM & $\begin{array}{l}\text { CF Model } \\
\text { (3): NPM }\end{array}$ & CF with PM \\
\hline AP1 & 0.2650 & 0.2650 & 0.2858 \\
\hline AP2 & 0.5457 & 0.5457 & 0.4002 \\
\hline AP3 & 0.5536 & 0.4332 & 0.3700 \\
\hline AP4 & 0.1867 & 0.1867 & 0.4736 \\
\hline AP5 & 0.5396 & 0.5396 & 0.3160 \\
\hline AP6 & 0.3885 & 0.3885 & 0.3288 \\
\hline AP7 & 0.0509 & 0.0509 & 0.4179 \\
\hline AP8 & 0.4695 & 0.5899 & 0.3477 \\
\hline AP9 & 0.2215 & 0.2215 & 0.2812 \\
\hline Balance Index ( $\mathbf{\beta})$ & $81.03 \%$ & $80.48 \%$ & $97.24 \%$ \\
\hline
\end{tabular}

Table 8. comparison between model (5) and model (6) (scenario 3)

\begin{tabular}{|l|l|l|}
\hline & $\begin{array}{l}\text { Initial Channel } \\
\text { Assignment }\end{array}$ & $\begin{array}{l}\text { Final Channel } \\
\text { Assignment }\end{array}$ \\
\hline AP1 & 4 & 6 \\
\hline AP2 & 9 & 1 \\
\hline AP3 & 1 & 11 \\
\hline AP4 & 11 & 8 \\
\hline AP5 & 1 & 11 \\
\hline AP6 & 11 & 4 \\
\hline AP7 & 6 & 6 \\
\hline AP8 & 11 & 8 \\
\hline AP9 & 6 & 11 \\
\hline Avg. SIR & $\mathbf{1 . 1 1}$ & $\mathbf{1 . 9 3}$ \\
\hline
\end{tabular}

Model (3) decreased the load at the MCAP $\left(A P_{8}\right)$ but increased it greatly on $A P_{3}$. Our PM algorithm distributed the load efficiently among APs (around 98\% balanced). The load distribution is achieved at the following transmitted power levels at the APs: $17 \mathrm{dBm}, 4 \mathrm{dBm}, 3$ $\mathrm{dBm}, 15 \mathrm{dBm}, 14 \mathrm{dBm}, 4 \mathrm{dBm}, 10 \mathrm{dBm}, 1$ $\mathrm{dBm}, 3 \mathrm{dBm}, 4 \mathrm{dBm}, 7 \mathrm{dBm}$, and $10 \mathrm{dBm}$, respectively.

It is noticed from the results in Table 10 that our algorithm assigned the same channels to some APs where there were no overlap- ping users, which caused the average SIR to improve greatly. The total SIR of the 60 users has improved from 0.4090 to 1.8508 (around $350 \%$ ). Figure 7 graphs the channels assigned to all 12 APs in scenario 4 and the AP positions followed in the previous four scenarios.

\section{CONCLUSION}

In this article, a new channel assignment algorithm has been proposed based on maximizing 
78 Int. J. of Interdisciplinary Telecommunications and Networking, 1(2), 66-81, April-June 2009

Table 9. Comparison between congestion factors (scenario 4)

\begin{tabular}{|l|l|l|l|}
\hline & CF :NPM & $\begin{array}{l}\text { CF Model } \\
\text { (3): NPM }\end{array}$ & CF with PM \\
\hline AP1 & 0.2622 & 0.2622 & 0.5199 \\
\hline AP2 & 0.8017 & 0.8017 & 0.6348 \\
\hline AP3 & 0.8243 & 0.9307 & 0.5417 \\
\hline AP4 & 0.3337 & 0.3337 & 0.6318 \\
\hline AP5 & 0.4391 & 0.4391 & 0.6161 \\
\hline AP6 & 0.6932 & 0.6932 & 0.6444 \\
\hline AP7 & 0.3969 & 0.3969 & 0.5023 \\
\hline AP8 & 0.9326 & 0.6930 & 0.7209 \\
\hline AP9 & 0.5591 & 0.6923 & 0.4058 \\
\hline AP10 & 0.7083 & 0.7083 & 0.5614 \\
\hline AP11 & 0.4816 & 0.4816 & 0.5003 \\
\hline AP12 & 0.5110 & 0.5110 & 0.5110 \\
\hline Balance Index $(\beta)$ & $89.08 \%$ & $89.80 \%$ & $97.92 \%$ \\
\hline
\end{tabular}

Table 10. Comparison between model (5) and model (6) ( scenario 4)

\begin{tabular}{|l|l|l|}
\hline & $\begin{array}{l}\text { Initial Channel } \\
\text { Assignment }\end{array}$ & $\begin{array}{l}\text { Final Channel As- } \\
\text { signment }\end{array}$ \\
\hline AP1 & 1 & 1 \\
\hline AP2 & 11 & 1 \\
\hline AP3 & 1 & 6 \\
\hline AP4 & 6 & 1 \\
\hline AP5 & 11 & 6 \\
\hline AP6 & 6 & 1 \\
\hline AP7 & 1 & 11 \\
\hline AP8 & 6 & 1 \\
\hline AP9 & 11 & 5 \\
\hline AP10 & 1 & 1 \\
\hline AP11 & 9 & 8 \\
\hline AP12 & 4 & 1 \\
\hline Avg. SIR & $\mathbf{0 . 4 0 9 0}$ & $\mathbf{1 . 8 5 0 8}$ \\
\hline
\end{tabular}

the SIR at the users in combination with a scheme that reduces congestion at the APs based on power management. The algorithm has been shown to provide superior results compared to previous work where channel assignment is based on minimizing interference between APs only, with no consideration given to users. We have shown that our proposed algorithm is scalable with different network sizes. The algorithm showed great improvement in the 
load distribution and average SIR. Channel assignment is better determined at the end of the balanced load state rather than keeping the initial channel assignment from the planning stage. This will improve the throughput of the network. Extensive computations suggest the 11-step algorithm leads toward more stable and better User-based channel assignment and load-balance

Since this is a prototype study, there is room for further research. Work is in progress to identify a more efficient heuristic algorithm to solve the optimization problems, and to include dynamic user behavior and mobility. The latter will determine how fast both algorithms can adapt to the dynamic environment. With these features in place, a more rigorous understanding of the convergence behavior of the algorithm can be obtained.

\section{REFERENCES}

Akl, R., \& Arepally, A. (2007). Dynamic Channel Assignment in IEEE 802.11 Networks. Proceedings of IEEE Portable 2007: International Conference on Portable Information Devices, Orlando, FL, USA, pp. 1-5.

Akl, R. \& Park, S. (2005). Optimal Access Point selection and Traffic Allocation in IEEE 802.11 Networks. Proceedings of 9th World Multiconference on Systemics, Cybernetics and Informatics (WMSCI 2005): Communication and Network Systems, Technologies and Applications, vol. 8, pp.75-79.

Balachandran, A., Bahl, P., Voelker, G. M. (2002). Hot-Spot Congestion Relief in Public-area Wireless Networks. Proceedings Fourth IEEE Workshop on Mobile Computing Systems and Applications, pp. 70-80.

Boulmalf, M., El-Sayed, H., \& Soufyane, A. (2005). Measured Throughput and SNR of IEEE 802.11g in a Small Enterprise Environment. $61^{\text {st }}$ IEEE Vehicular Technology Conference (VTC'05), UAE, Vol. 2, pp. 1333-1337.

Chiu, D. \& Jain, R. (1989). Analysis of the Increase and Decrease Algorithms for Congestion Avoidance in Computer Networks. Journal of Computer Networks and ISDN, vol. 17, pp. 1-14.
Eisenblätter, A., Geerdes, H. F., \& Siomina, I. (2007). Integrated Access Point Placement and Channel Assignment for Wireless LANs in an Indoor Office Environment. 8th IEEE Intl. Symposium on a World of Wireless, Mobile and Multimedia Networks.

Fang, C. \& Low, C. P. (2004). Efficient Algorithms for the Load-Balanced Demand Point Assignment Problem in Large-Scale WLAN. $12^{\text {th }}$ IEEE Conference on Networks, Vol. 1, pp.196-200.

Haidar, M., Akl, R., Al-Rizzo, H. M., Chan, Y., \& Adada, R. (2007). Optimal Load Distribution in Large Scale WLAN Networks Utilizing a Power Management Algorithm. Proceedings of IEEE Sarnoff Symposium.

Haidar, M., Al-Rizzo, H. M., Akl, R., \& Chan, Y. (2007).Channel Assignment and Load Distribution in a Power-managed WLAN. Proceedings of 18th Annual IEEE International Symposium on Personal, Indoor and Mobile Radio Communications, Athens, Greece.

Kulkrani, P. \& Shenoy, P. (2004). Exploiting Overlap for Provisioning of Access Points in Wireless Networks (Tech. Report). Boston, Massachusetts: University of Massachusetts.

Lee, Y., Kim, K., Choi, Y. (2002). Optimization of AP placement and Channel Assignment in Wireless LANs. LCN 2002. 27 th Annual IEEE Conference on Local Computer Networks, pp. 831-836.

Lei, J., Yates, R., Greenstein, L., \& Liu, H. (2005). Wireless Link SNR Mapping Onto an Indoor Testbed. Proceedings of IEEE Tridentcom 2005, Trento, Italy.

Mishra, A., Banerjee, S., \& Arbaugh, W. (2005). Weighted Coloring Based Channel Assignment for WLANs. ACM SIGMOBILE Mobile Computing and Communications Review, vol.9, pp.19-31.

Mishra, A., Brik, V., Banarjee, S., Srinivasan, A., \& Arbaugh, W. (2006). A Client-Driven Approach for Channel Management in Wireless LANs. $25^{\text {th }}$ International Conference on Computer Communications, pp.1-12.

Papanikos, I. \& Logothetis, M. (2001). A Study on Dynamic Load Balance for IEEE 802.11b Wireless LAN. Proc. 8th International Conference on Advances in Communication \& Control, COMCON 8 , Rethymna, Crete/Greece. 
Velayos, H., Aleo, V., \& Karlsson (2004). Load Balancing in Overlapping Wireless LAN Cells. Proceedings of IEEE ICC 2004, Paris, France.

Mohamad Haidar received his BS degree in electrical engineering from the Lebanese American University, Byblos, Lebanon in 2000. The MS degree was received from Roosevelt University in telecommunications, Chicago, IL, in 2002 and PhD in applied science from the University of Arkansas at Little Rock (UALR), Little Rock, AR, in 2007. His major field of research is in wireless networking and communications. Previously, he worked as a development engineer at IBM, Durham, NC, teacher assistant at UALR, Little Rock, $A R$, and WiMesh/WiMax RF engineer analyst on a UALR-Alltel-Motorola project. His research interests are load balancing, channel assignment, MAC and PHY layers design in WLAN, cognitive wireless mesh networks, ad hoc networks, and WiMax environments.

Hussain Al-Rizzo received his BSc in electronics and communications (1979) (High Honors), postgraduate diploma in electronics and communications (1981) (high honors) and MSc in microwave communication systems (1983) from the University of Mosul, Mosul, Iraq. From May 1983 to October 1987 he was working with the Electromagnetic Wave Propagation Department, Space and Astronomy Research Center, Scientific Research Council, Baghdad, Iraq. On December, 1987, he joined the Radiating Systems Research Laboratory, Electrical and Computer Engineering Department, University of New Brunswick, Fredericton, NB, Canada where he obtained his PhD (1992) in electromagnetic, wireless communications, and the global positioning system. For his various academic achievements he won the nomination by the University of New Brunswick as the best doctoral graduate in science and engineering. Since 2000, he joined the Systems Engineering Department, University Arkansas at Little Rock where he is currently a tenured associate professor. He has published over 25 peer-reviewed journal papers and 54 conference presentations. His research areas include implantable antennas and wireless systems, smart antennas, WLAN deployment and load balancing, electromagnetic wave scattering by complex objects, design, modeling and testing of high-power microwave applicators, design and analysis of microstrip antennas for mobile radio systems, precipitation effects on terrestrial and satellite frequency re-use communication systems, field operation of NAVSTAR GPS receivers, data processing, and accuracy assessment, effects of the ionosphere, troposphere and multipath on code and carrier-beat phase GPS observations and the development of novel hybrid Cartesian/cylindrical FD-TD models for passive microwave components.

Yupo Chan received his PhD from Massachusetts Institute of Technology in 1972. After 28 years of post-doctoral experience in industry, universities and government, he became the founding chair of the Department of Systems Engineering at the University of Arkansas at Little Rock (UALR) Donaghey College of Information Science and Systems Engineering (CyberCollege) in 2000. Before coming to the UALR CyberCollege, Chan worked at The Air Force Institute of Technology, Washington State University, the State University of New York at Stony Brook, Pennsylvania State University, and Kates, Peat Marwick. Additionally, he was a congressional fellow in the Office of Technology Assessment in Washington, DC. Chan's training and research focus on transportation systems, telecommunications, networks and combinatorial optimization, multi-criteria decision-making and spatial-temporal information. Chan has published numerous books and monographs, including Location Theory and Decision Analysis (Thomson/South-Western); Location, Transportation, and Land-Use: Modeling Spatial-temporal Information (Springer); Urban Planning and Development Applications of GIS (ASCE Press).

Robert Akl received the BS degree in computer science from Washington University in St. Louis, in 1994, and the BS, MS and DSc degrees in electrical engineering in 1994, 1996, and 2000, respectively. He also

Copyright (C 2009, IGI Global. Copying or distributing in print or electronic forms without written permission of IGI Global is prohibited. 
received the Dual Degree Engineering Outstanding Senior Award from Washington University in 1993. He is a senior member of IEEE. Akl is currently an associate professor at the University of North Texas, Department of Computer Science and Engineering. In 2002, he was an assistant professor at the University of New Orleans, Department of Electrical and Computer Engineering. From October 2000 to December 2001, he was a senior systems engineer at Comspace Corporation, Coppell, TX. His research interests include wireless communication and network design and optimization. 